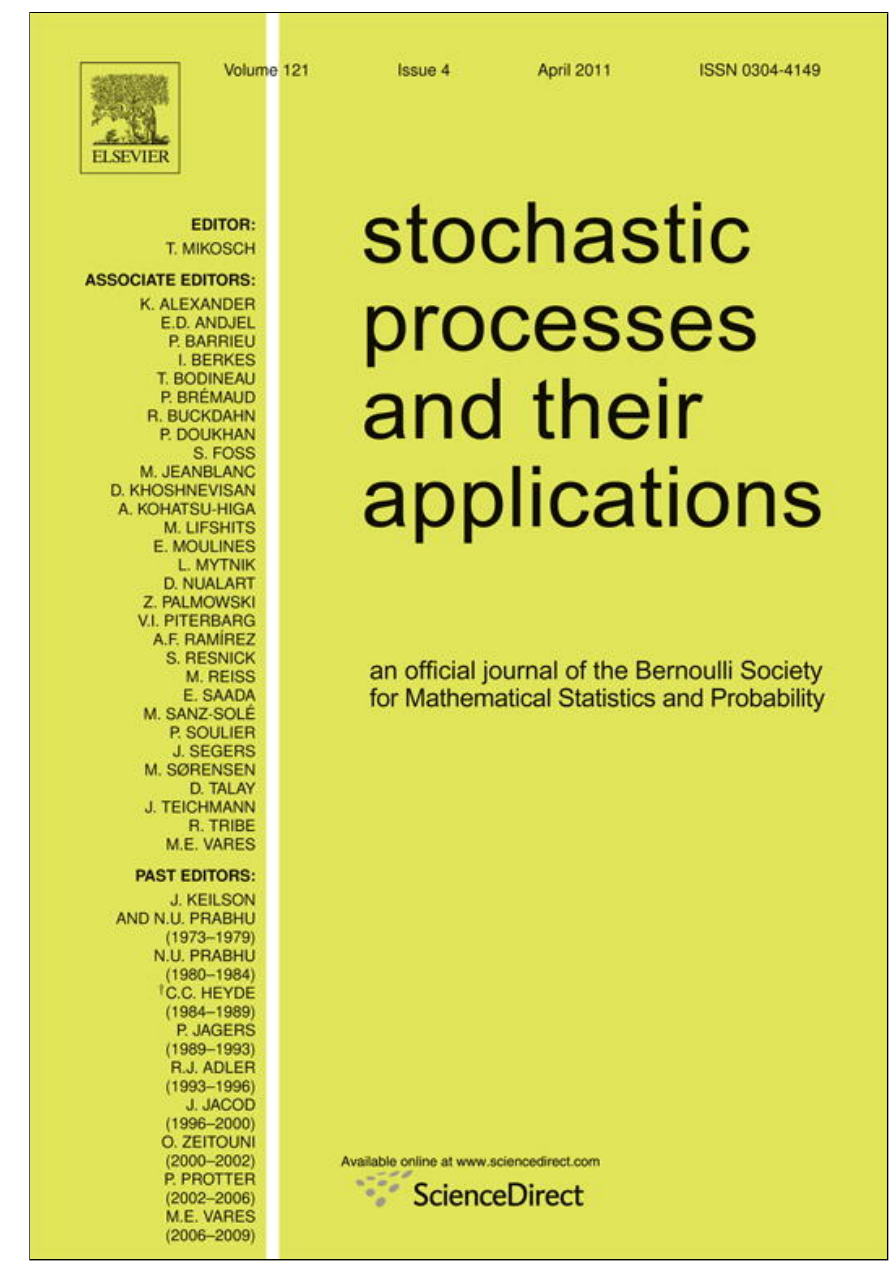

This article appeared in a journal published by Elsevier. The attached copy is furnished to the author for internal non-commercial research and education use, including for instruction at the authors institution and sharing with colleagues.

Other uses, including reproduction and distribution, or selling or licensing copies, or posting to personal, institutional or third party websites are prohibited.

In most cases authors are permitted to post their version of the article (e.g. in Word or Tex form) to their personal website or institutional repository. Authors requiring further information regarding Elsevier's archiving and manuscript policies are encouraged to visit:

http://www.elsevier.com/copyright 


\title{
Quantitative Breuer-Major theorems
}

\author{
Ivan Nourdin $^{\mathrm{a}}$, Giovanni Peccati ${ }^{\mathrm{b}}$, Mark Podolskij $^{\mathrm{c}, *}$ \\ a Laboratoire de Probabilités et Modèles Aléatoires, Université Pierre et Marie Curie, Boîte courrier 188, 4 Place \\ Jussieu, 75252 Paris Cedex 5, France \\ ${ }^{\mathrm{b}}$ Faculté des Sciences, de la Technologie et de la Communication; UR en Mathématiques. 6, rue Richard \\ Coudenhove-Kalergi, L-1359, Luxembourg \\ ${ }^{\mathrm{c}}$ Department of Mathematics, ETH Zürich, HG G32.2, 8092 Zürich, Switzerland
}

Received 12 July 2010; received in revised form 3 December 2010; accepted 15 December 2010

Available online 22 December 2010

\begin{abstract}
We consider sequences of random variables of the type $S_{n}=n^{-1 / 2} \sum_{k=1}^{n}\left\{f\left(X_{k}\right)-E\left[f\left(X_{k}\right)\right]\right\}, n \geq 1$, where $X=\left(X_{k}\right)_{k \in \mathbb{Z}}$ is a $d$-dimensional Gaussian process and $f: \mathbb{R}^{d} \rightarrow \mathbb{R}$ is a measurable function. It is known that, under certain conditions on $f$ and the covariance function $r$ of $X, S_{n}$ converges in distribution to a normal variable $S$. In the present paper we derive several explicit upper bounds for quantities of the type $\left|\mathbb{E}\left[h\left(S_{n}\right)\right]-\mathbb{E}[h(S)]\right|$, where $h$ is a sufficiently smooth test function. Our methods are based on Malliavin calculus, on interpolation techniques and on the Stein's method for normal approximation. The bounds deduced in our paper depend only on $\operatorname{Var}\left[f\left(X_{1}\right)\right]$ and on simple infinite series involving the components of $r$. In particular, our results generalize and refine some classic CLTs given by Breuer and Major, Giraitis and Surgailis, and Arcones, concerning the normal approximation of partial sums associated with Gaussiansubordinated time series.
\end{abstract}

(C) 2010 Elsevier B.V. All rights reserved.

MSC: 60F05; 60H05; 60G15; 60H07

Keywords: Berry-Esseen bounds; Breuer-Major central limit theorems; Gaussian processes; Interpolation; Malliavin calculus; Stein's method

\footnotetext{
* Corresponding author.

E-mail addresses: ivan.nourdin@upmc.fr (I. Nourdin), giovanni.peccati@gmail.com (G. Peccati), mpodolskij@creates.au.dk, mark.podolskij@math.ethz.ch (M. Podolskij).
} 


\section{Introduction}

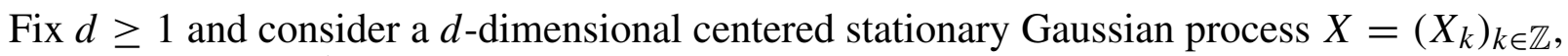
$X_{k}=\left(X_{k}^{(1)}, \ldots, X_{k}^{(d)}\right)$, defined on a probability space $(\Omega, \mathcal{F}, \mathbb{P})$. For any $1 \leq i, l \leq d$ and $j \in \mathbb{Z}$, we denote by

$$
r^{(i, l)}(j)=\mathbb{E}\left[X_{1}^{(i)} X_{1+j}^{(l)}\right],
$$

the covariance of $X_{1}^{(i)}$ and $X_{1+j}^{(l)}$. Let $f: \mathbb{R}^{d} \rightarrow \mathbb{R}$ be a measurable function and write

$$
S_{n}=\frac{1}{\sqrt{n}} \sum_{k=1}^{n}\left\{f\left(X_{k}\right)-\mathbb{E}\left[f\left(X_{k}\right)\right]\right\}, \quad n \geq 1,
$$

to indicate the sequence of normalized partial sums associated with the subordinated process $k \mapsto f\left(X_{k}\right)$. One crucial problem in Gaussian analysis is the following:

Problem P. Find conditions on $f$ and on the covariance $r$ in order to have that, as $n \rightarrow \infty, S_{n}$ converges in distribution to a Gaussian random variable.

Although easily stated, Problem P is in fact quite subtle. For instance, as observed e.g. in [8, p. 429], it is in general not possible to deduce a solution to Problem $\mathrm{P}$ by using standard central limit results for dependent random variables (for instance, by applying techniques based on mixing). More to the point, slight variations in the form of $f$ and $r$ may imply that either the normalization by the factor $n^{1 / 2}$ is inappropriate, or the limiting distribution is not Gaussian (or both apply): see Dobrushin and Major [15], Rosenblatt [31] and Taqqu [36,37] for several classic results connected with this phenomenon, as well as Breton and Nourdin [7] for recent developments.

It turns out that an elegant solution to Problem $\mathrm{P}$ can be deduced by using the notion of Hermite rank. Recall that the function $f$ is said to have Hermite rank equal to $q$ with respect to $X$, where $q \geq 1$ is an integer, if (a) $\mathbb{E}\left[(f(X)-\mathbb{E}[f(X)]) p_{m}(X)\right]=0$ for every polynomial $p_{m}$ (on $\mathbb{R}^{d}$ ) of degree $m \leq q-1$; and (b) there exists a polynomial $p_{q}$ of degree $q$ such that $\mathbb{E}\left[(f(X)-E[f(X)]) p_{q}(X)\right] \neq 0$.

Then, one has the following well-known statement:

Theorem 1.1 (Breuer-Major Theorem for Stationary Vectors). Let $\mathbb{E}\left[f^{2}\left(X_{1}\right)\right]<\infty$, and assume that the function $f$ has Hermite rank equal to $q \geq 1$. Suppose that

$$
\sum_{j \in \mathbb{Z}}\left|r^{(i, l)}(j)\right|^{q}<\infty, \quad \forall i, l \in\{1, \ldots, d\}
$$

Then $\sigma^{2}:=\operatorname{Var}\left[f^{2}\left(X_{1}\right)\right]+2 \sum_{k=1}^{\infty} \operatorname{Cov}\left[f\left(X_{1}\right), f\left(X_{1+k}\right)\right]$ is well-defined, and belongs to $[0, \infty)$. Moreover, one has that

$$
S_{n} \stackrel{d}{\longrightarrow} S \sim N\left(0, \sigma^{2}\right),
$$

where $N\left(0, \sigma^{2}\right)$ indicates a centered Gaussian distribution with variance $\sigma^{2}$, and $\stackrel{d}{\longrightarrow}$ stands for convergence in distribution.

In the case $d=1$, Theorem 1.1 was first proved by Breuer and Major in [8], whereas Theorem 4 in Arcones [2] proves the statement for a general $d$ (both proofs in [2,8] are based on the method of cumulants and diagram formulae — see e.g. [29,34]). The reader is referred 
to Sun [33] for an early statement in the case of a Hermite rank equal to 2, and to Giraitis and Surgailis [17] for some continuous-time analogues of Theorem 1.1. Note that any central limit result involving Hermite ranks and series of covariance coefficients is customarily called a 'Breuer-Major theorem', in honor of the seminal paper [8].

Theorem 1.1 and its variations have served as fundamental tools for Gaussian approximations in an impressive number of applications, of which we provide a representative (recent) sample: renormalization of fractional diffusions [1], power variations of Gaussian and Gaussian-related continuous-time processes [3,4,14,20,24], Gaussian fluctuations of heat-type equations [5], estimation of Hurst parameters of fractional processes [9,12,13], unit-root problems in econometrics [10], empirical processes of long-memory time series [22], level functionals of stationary Gaussian fields [19], variations of multifractal random walks [21], and stochastic programming [38]. See also Surgailis [34] for a survey of some earlier uses of Breuer-Major criteria.

Despite this variety of applications, until recently the only available techniques for proving results such as Theorem 1.1 were those based on combinatorial cumulants/diagrams computations. These techniques are quite effective and flexible (see e.g. [23,32] for further instances of their applicability), but suffer from a fundamental drawback, namely they do not allow us to deduce Berry-Esseen relations of the type

$$
\left|\mathbb{E}\left[h\left(S_{n}\right)\right]-\mathbb{E}[h(S)]\right| \leq \varphi(n), \quad n \geq 1,
$$

where $h$ is a suitable test function, and $\varphi(n) \rightarrow 0$ as $n \rightarrow \infty$. An upper bound such as (1.5) quantifies the error that one makes when replacing $S_{n}$ with $S$ for a fixed $n$.

In [25, Section 4], the first two authors of this paper proved that one can combine Malliavin calculus (see e.g. [28]) and Stein's method (see e.g. [11]) to obtain relations such as (1.5) (for some explicit $\varphi(n)$ ) in the case where: (i) $d=1$, (ii) $f=H_{q}$ is a Hermite polynomial of degree $q \geq 2$ (and thus has Hermite rank equal to $q$ ), (iii) $X$ is obtained from the increments of a fractional Brownian motion of Hurst index $H<1-(2 q)^{-1}$, and (iv) $h$ is either an indicator of a Borel set or a Lipschitz function. Since under (iii) one has that $|r(j)| \sim j^{2 H-2}$, these findings allow us to recover a very special case of Theorem 1.1 (see Example 2.5 below for more details on this point).

The aim of the present work is to extend the techniques initiated in [25] in order to deduce several complete quantitative Breuer-Major theorems, that is, statements providing explicit upper bounds such as (1.5) for any choice of $f$ and $r$ satisfying the assumptions of Theorem 1.1. We stress now that we will not require that the functions $f$ enjoy any additional smoothness property, so our results represent a genuine extension of the findings by Breuer and Major and by Arcones.

As anticipated, our techniques are based on the use of Malliavin operators on a Gaussian space, that we combine both with Stein's method and with an interpolation technique (already applied in [27,30]) which is reminiscent of the 'smart path method' in spin glasses — see e.g. Talagrand [35]. In particular, the use of Stein's method allows us to deal with functions $h$ that are either Lipschitz or indicators of the type $h=\mathbf{1}_{(-\infty, z]}$, whereas the use of interpolations requires test functions that are twice differentiable and with bounded derivatives. Note that this implies that the convergence (1.4) does indeed take place in the stronger topologies of the Kolmogorov and Wasserstein distances.

The rest of the paper is organized as follows. Section 2 contains the statements of our main results, some examples and applications. Section 3 presents some notions and results that are needed to prove our main Theorem 2.1. Section 4 is devoted to proofs. 


\section{Statement of the main results}

We keep the assumptions and notation of the previous section. For the sake of notational simplicity, in the following we shall assume that $\mathbb{E}\left[f\left(X_{1}\right)\right]=0$. Also, we shall assume that $X_{1} \sim$ $N_{d}\left(0, I_{d}\right)$, implying that $X_{k}^{(1)}, \ldots, X_{k}^{(d)}$ are independent $N(0,1)$ random variables for all $k \in \mathbb{Z}$. Note that this last assumption is not restrictive: indeed, by reduction of variables and at the cost of possibly decreasing the value of $d$, we may always assume that $X_{k} \sim N_{d}(0, \Sigma)$ for an invertible matrix $\Sigma \in \mathbb{R}^{d \times d}$, and by a linear transformation we can further restrict ourselves to the case $X_{k} \sim N_{d}\left(0, I_{d}\right)$. Observe that, if $f(x)$ has Hermite rank $q$ with respect to $X_{1}$, then $f(L x)$ has the same Hermite rank $q$ with respect to $L^{-1} X_{1}$, for every invertible matrix $L$ - see also [2, p. 2249].

Now, let $\Lambda$ denote the set of all vectors $\alpha=\left(\alpha_{1}, \ldots, \alpha_{d}\right)$ with $\alpha_{i} \in \mathbb{N} \cup\{0\}$. For any multi-index $\alpha \in \Lambda$, we introduce the notation $|\alpha|=\sum_{i=1}^{d} \alpha_{i}$ and $\alpha$ ! $=\prod_{i=1}^{d} \alpha_{i} !$. When $\mathbb{E}\left[f^{2}\left(X_{1}\right)\right]<\infty$, the function $f$ possesses the unique Hermite expansion

$$
f(x)=\sum_{\alpha \in \Lambda} a_{\alpha} \prod_{i=1}^{d} H_{\alpha_{i}}\left(x_{i}\right), \quad a_{\alpha}=(\alpha !)^{-1} \mathbb{E}\left[f\left(X_{1}\right) \prod_{i=1}^{d} H_{\alpha_{i}}\left(X_{1}^{(i)}\right)\right],
$$

where $\left(H_{j}\right)_{j \geq 0}$ is the sequence of Hermite polynomials, recursively defined as follows: $H_{0}=1$, and

$$
H_{j}=\delta H_{j-1}, \quad j \geq 1,
$$

where $\delta f(x)=x f(x)-f^{\prime}(x)$ (for instance: $H_{1}(x)=x, H_{2}(x)=x^{2}-1$, and so on). When $f$ has Hermite rank $q \geq 1$ we introduce the decomposition

$$
f(x)=\sum_{m=q}^{\infty} f_{m}(x), \quad f_{m}(x)=\sum_{\alpha \in \Lambda:|\alpha|=m} a_{\alpha} \prod_{i=1}^{d} H_{\alpha_{i}}\left(x_{i}\right) .
$$

Notation. Fix a function $f$ such that $\mathbb{E}\left[f^{2}\left(X_{1}\right)\right]<\infty$ and $f$ has Hermite rank $q \geq 1$. Our main results are expressed in terms of the following collection of coefficients (2.8)-(2.12):

$$
\begin{aligned}
& \theta(j)=\max _{1 \leq i, l \leq d}\left|r^{(i, l)}(j)\right| \\
& K=\inf _{k \in \mathbb{N}}\left\{\theta(j) \leq d^{-1}, \forall|j| \geq k\right\} \quad(\text { with } \inf \emptyset=\infty), \\
& \theta=\sum_{j \in \mathbb{Z}} \theta(j)^{q}, \\
& \sigma_{m}^{2}=\mathbb{E}\left[f_{m}^{2}\left(X_{1}\right)\right]+2 \sum_{k=1}^{\infty} \mathbb{E}\left[f_{m}\left(X_{1}\right) f_{m}\left(X_{1+k}\right)\right] \quad(\text { for } m \geq q), \\
& \left.\gamma_{n, m, e}=\sqrt{2 \theta n^{-1} \sum_{|j| \leq n} \theta(j)^{e} \sum_{|j| \leq n} \theta(j)^{m-e}} \quad \text { (for } m \geq q \text { and } 1 \leq e \leq m-1\right) .
\end{aligned}
$$

The coefficients $\theta(j), K, \theta, \sigma_{m}^{2}$ and $\gamma_{n, m, e}$ will also be combined into the following expressions (2.13)-(2.17):

$$
A_{1, n}=\frac{\mathbb{E}\left[f^{2}\left(X_{1}\right)\right]}{2}\left[\frac{2 K^{2}}{n}+d^{q}\left(\sum_{|j| \leq n} \theta(j)^{q} \frac{|j|}{n}+\sum_{|j|>n} \theta(j)^{q}\right)\right]
$$




$$
\begin{aligned}
A_{2, N}= & 2\left(2 K+d^{q} \theta\right) \sqrt{\mathbb{E}\left[f^{2}\left(X_{1}\right)\right] \sum_{m=N+1}^{\infty} \mathbb{E}\left[f_{m}^{2}\left(X_{1}\right)\right]} \\
A_{3, n, N}= & \frac{\mathbb{E}\left[f^{2}\left(X_{1}\right)\right]}{2} \sum_{m=q}^{N}\left(\frac{d^{m}}{m m !} \sum_{l=1}^{m-1} l l !\left(\begin{array}{c}
m \\
l
\end{array}\right)^{2} \sqrt{(2 m-2 l) !} \gamma_{n, m, l}\right) \\
A_{4, n, N}= & \frac{\mathbb{E}\left[f^{2}\left(X_{1}\right)\right]\left(2 K+d^{q} \theta\right)^{1 / 2}}{2} \\
& \times \sum_{q \leq p<s \leq N} d^{s / 2} \sqrt{\frac{p !}{s !}} \frac{p+s}{p}\left(\begin{array}{l}
s-1 \\
p-1
\end{array}\right) \sqrt{(s-p) !} \gamma_{n, s, s-p}^{1 / 2} \\
A_{5, n, N}= & \frac{\mathbb{E}\left[f^{2}\left(X_{1}\right)\right]}{2 \sqrt{2}} \sum_{q \leq p<s \leq N}(p+s) \sum_{l=1}^{p-1}(l-1) !\left(\begin{array}{l}
p-1 \\
l-1
\end{array}\right)\left(\begin{array}{l}
s-1 \\
l-1
\end{array}\right) \sqrt{(p+s-2 l) !} \\
& \times\left(\frac{d^{s}}{s !} \gamma_{n, s, s-l}+\frac{d^{p}}{p !} \gamma_{n, p, p-l}\right)
\end{aligned}
$$

Note that the coefficients $K, \theta$ and $\sigma_{m}$ can in general be infinite, and also that, if $\mathbb{E}\left[f\left(X_{1}\right)^{2}\right]<\infty$, if $f$ has Hermite rank $q$, and if (1.3) is in order, then

$$
\sigma^{2}=\sum_{m=q}^{\infty} \sigma_{m}^{2}<\infty
$$

where $\sigma^{2}$ is defined in Theorem 1.1.

The next statement, which is the main result of the paper, asserts that the quantities defined above can be used to write explicit bounds of the type (1.5).

Theorem 2.1 (Quantitative Breuer-Major Theorem). Let the notation and assumptions of this section prevail (in particular, $\mathbb{E}\left[f\left(X_{1}\right)\right]=0$ and $X_{1} \sim N_{d}\left(0, I_{d}\right)$ ), and assume that the conditions of Theorem 1.1 are satisfied. Then, the coefficients appearing in formulae (2.8)(2.17) are all finite. Moreover, the following three bounds are in order.

(1) For any function $h \in C^{2}(\mathbb{R})$ (that is, $h$ is twice continuously differentiable) with bounded second derivative, and for every $n>K$,

$$
\left|\mathbb{E}\left[h\left(S_{n}\right)\right]-\mathbb{E}[h(S)]\right| \leq\left\|h^{\prime \prime}\right\|_{\infty}\left(A_{1, n}+\inf _{N \geq q}\left\{A_{2, N}+A_{3, n, N}+A_{4, n, N}+A_{5, n, N}\right\}\right) .
$$

(2) For any Lipschitz function $h$, and for every $n>K$,

$$
\begin{aligned}
& \left|\mathbb{E}\left[h\left(S_{n}\right)\right]-\mathbb{E}[h(S)]\right| \leq \frac{\left\|h^{\prime}\right\|_{\infty}}{2}\left\{A_{2, n}\left[\frac{1}{\sigma}+\frac{1}{\sqrt{\left(2 K+d^{q} \theta\right) \mathbb{E}\left[f^{2}\left(X_{1}\right)\right]}}\right]\right. \\
& \left.\quad+4 \inf _{N \geq q} \frac{A_{1, n}+A_{3, n, N}+A_{4, n, N}+A_{5, n, N}}{\sqrt{\sum_{m=q}^{N} \sigma_{m}^{2}}}\right\}
\end{aligned}
$$

(3) For any $z \in \mathbb{R}$, and for every $n>K$, 


$$
\begin{aligned}
& \left|\left[\mathbb{P}\left(S_{n} \leq z\right)\right]-\mathbb{P}(S \leq z)\right| \leq \frac{\sqrt{2}}{\sigma} \\
& \quad \times \sqrt{A_{2, n}\left[\frac{1}{\sigma}+\frac{1}{\sqrt{\left(2 K+d^{q} \theta\right) \mathbb{E}\left[f^{2}\left(X_{1}\right)\right]}}\right]+4 \inf _{N \geq q} \frac{A_{1, n}+A_{3, n, N}+A_{4, n, N}+A_{5, n, N}}{\sqrt{\sum_{m=q}^{N} \sigma_{m}^{2}}}} .
\end{aligned}
$$

We will now demonstrate that Theorem 2.1 implies a stronger version of Theorem 1.1, namely that the convergence (1.4) takes place with respect to topologies that are stronger than the one of convergence in distribution. To prove this claim, we need to show in particular that, under the assumptions of Theorem 1.1, $\gamma_{n, m, e} \rightarrow 0$ as $n \rightarrow \infty$ for any choice of $m \geq q$ and $1 \leq e \leq m-1$. This is a consequence of the next Lemma 2.2. In what follows, given positive sequences $b_{n}, c_{n}$, $n \geq 1$, we shall write $b_{n} \lesssim c_{n}$ whenever $b_{n} / c_{n}$ is bounded, and $b_{n} \sim c_{n}$ if $b_{n} \lesssim c_{n}$ and $c_{n} \lesssim b_{n}$.

Lemma 2.2. Let $\left(a_{k}\right)_{k \in \mathbb{Z}}$ be a sequence of positive real numbers such that $\sum_{k \in \mathbb{Z}} a_{k}^{m}<\infty$ for some $m \in \mathbb{N}$. If $1 \leq e \leq m-1$, then

$$
n^{-1+\frac{e}{m}} \sum_{|k| \leq n} a_{k}^{e} \rightarrow 0
$$

Proof. Fix $\delta \in(0,1)$, and decompose the sum as $\sum_{k=1}^{n}=\sum_{k=1}^{[n \delta]}+\sum_{k=[n \delta]+1}^{n}$. By the Hölder inequality we obtain (recall that $\sum_{k=1}^{\infty} a_{k}^{m}$ is finite)

$$
n^{-1+e / m} \sum_{k=1}^{[n \delta]} a_{k}^{e} \leq n^{-1+e / m}(n \delta)^{1-e / m}\left(\sum_{k=1}^{\infty} a_{k}^{m}\right)^{e / m} \leq c \delta^{1-e / m},
$$

where $c$ is some constant, as well as

$$
n^{-1+e / m} \sum_{k=[n \delta]+1}^{n} a_{k}^{e} \leq\left(\sum_{k=[n \delta]+1}^{n} a_{k}^{m}\right)^{e / m} .
$$

The first term converges to 0 as $\delta$ goes to zero (because $1 \leq e \leq m-1$ ), and the second also converges to 0 for fixed $\delta$ and $n \rightarrow \infty$. This proves the claim.

Now recall that, if $X, Y$ are two real-valued random variables, then the Kolmogorov distance between the law of $X$ and the law of $Y$ is given by

$$
d_{K o l}(X, Y)=\sup _{z \in \mathbb{R}}|\mathbb{P}(X \leq z)-\mathbb{P}(Y \leq z)| .
$$

If $\mathbb{E}|X|, \mathbb{E}|Y|<\infty$, one can also meaningfully define the Wasserstein distance

$$
d_{W}(X, Y)=\sup _{f \in \operatorname{Lip}(1)}|\mathbb{E}[f(X)]-\mathbb{E}[f(Y)]|,
$$

where Lip(1) indicates the collection of all Lipschitz functions with Lipschitz constant $\leq 1$. Finally, if $X, Y$ have finite second moments, for every constant $C>0$ one can define the distance

$$
d_{C}(X, Y)=\sup _{f \in \mathscr{D}_{C}^{2}}|\mathbb{E}[f(X)]-\mathbb{E}[f(Y)]|
$$


where $\mathscr{D}_{C}^{2}$ stands for the class of all functions that are twice continuously differentiable and have a second derivative bounded by $C$. Note that the topologies induced by $d_{K o l}, d_{W}$ and $d_{C}$, on the probability measures on $\mathbb{R}$, are strictly stronger than the topology of convergence in distribution (see e.g. [16, Ch. 11]).

The next consequence of Theorem 2.1 provides the announced refinement of Theorem 1.1.

Corollary 2.3 (Breuer-Major, Strong Version). Let the notation and assumptions of this section prevail (in particular, $\mathbb{E}\left[f\left(X_{1}\right)\right]=0$ and $X_{1} \sim N_{d}\left(0, I_{d}\right)$ ), and assume that the conditions of Theorem 1.1 are satisfied. Then, the convergence (1.4) takes place with respect to the three distances $d_{K o l}, d_{W}$ and $d_{C}($ for all $C>0$ ), namely

$$
\lim _{n \rightarrow \infty} d_{K o l}\left(S_{n}, S\right)=\lim _{n \rightarrow \infty} d_{W}\left(S_{n}, S\right)=\lim _{n \rightarrow \infty} d_{C}\left(S_{n}, S\right)=0
$$

Proof. Under the assumptions of Theorem 1.1, one has that $A_{1, n} \rightarrow 0$ as $n \rightarrow \infty$ (because $\theta<\infty, \sum_{|j| \leq n} \theta(j)^{q} \frac{|j|}{n} \rightarrow 0$ as $n \rightarrow \infty$ by bounded convergence). On the other hand, because of (2.18) and since $\mathbb{E}\left[f^{2}\left(X_{1}\right)\right]<\infty$, one has that $A_{2, N} \rightarrow 0$ as $N \rightarrow \infty$. Moreover, since $\gamma_{n, m, e} \rightarrow 0$ for any $m \geq q$ and $1 \leq e \leq m-1$ (due to Lemma 2.2), one has that $A_{j, n, N} \rightarrow 0, j=$ $3,4,5$, for any fixed $N$ as $n \rightarrow \infty$. We deduce that $\inf _{N \geq q}\left\{A_{2, N}+A_{3, n, N}+A_{4, n, N}+A_{5, n, N}\right\} \rightarrow 0$ as $n \rightarrow \infty$. To conclude the proof, it remains to apply (2.19)-(2.21).

Next, we present a simplified version of Theorem 2.1 for $d=1$ and $f=H_{q}$, where $H_{q}$ is the $q$ th Hermite polynomial. Notice that in this case $K=0$ and the constants $A_{1, n}$ and $A_{3, n, N}=A_{3, n}$ are given by

$$
\begin{aligned}
& A_{1, n}=\frac{q !}{2} \theta\left(\sum_{|j| \leq n}|r(j)|^{q} \frac{|j|}{n}+\sum_{|j|>n}|r(j)|^{q}\right), \\
& A_{3, n}=\frac{1}{2 q} \sum_{l=1}^{q-1} l l !\left(\begin{array}{l}
q \\
l
\end{array}\right)^{2} \sqrt{(2 q-2 l) !} \gamma_{n, q, l},
\end{aligned}
$$

with $\gamma_{n, q, l}$ defined by (2.12).

Corollary 2.4 (Hermite Subordination). Assume that $d=1, f=H_{q}$ and $\sum_{j \in \mathbb{Z}}|r(j)|^{q}<\infty$, and let $A_{1, n}, A_{3, n}$ be given by (2.25)-(2.26).

(1) For any function $h \in C^{2}(\mathbb{R})$ with bounded second derivative it holds that

$$
\left|\mathbb{E}\left[h\left(S_{n}\right)\right]-\mathbb{E}[h(S)]\right| \leq\left\|h^{\prime \prime}\right\|_{\infty}\left(A_{1, n}+A_{3, n}\right) .
$$

(2) For any Lipschitz function $h$ it holds that

$$
\left|\mathbb{E}\left[h\left(S_{n}\right)\right]-\mathbb{E}[h(S)]\right| \leq \frac{2\left\|h^{\prime}\right\|_{\infty}}{\sigma}\left(A_{1, n}+A_{3, n}\right) .
$$

(3) For any $z \in \mathbb{R}$ it holds that

$$
\left|\left[\mathbb{P}\left(S_{n} \leq z\right)\right]-\mathbb{P}(S \leq z)\right| \leq \frac{2\left\|s_{z}^{\prime}\right\|_{\infty}}{\sigma}\left(A_{1, n}+A_{3, n}\right),
$$

where $s_{z}$ is the solution of the Stein's equation associated with the function $h(x)=$ $1_{(-\infty, z]}(x)$, i.e. $s_{z}$ solves the differential equation

$$
1_{(-\infty, z]}(x)-\Phi(z)=s_{z}^{\prime}(x)-x s_{z}(x), \quad x \in \mathbb{R},
$$


with $\Phi$ being the distribution function of $N(0,1)$. Furthermore, we have that $\left\|s_{z}^{\prime}\right\|_{\infty} \leq 1$ for all $z \in \mathbb{R}$.

Proof. From Theorem 3.1 in [25] and Theorem 3.2 in Section 3.3 we obtain the estimate

$$
\left|\mathbb{E}\left[h\left(S_{n}\right)\right]-\mathbb{E}[h(S)]\right| \leq c_{h} \mathbb{E}\left|\sigma^{2}-\left\langle D F,-D L^{-1} F\right\rangle_{\mathfrak{H}}\right|,
$$

where $c_{h}=\frac{\left\|h^{\prime \prime}\right\|_{\infty}}{2}$ in (1), $c_{h}=\frac{\left\|h^{\prime}\right\|_{\infty}}{\sigma}$ in (2) and $c_{h}=\frac{\left\|s_{z}^{\prime}\right\|_{\infty}}{\sigma}$ in (3). We readily deduce the assertion since $\mathbb{E}\left|\sigma^{2}-\left\langle D F,-D L^{-1} F\right\rangle_{\mathfrak{H}}\right| \leq 2\left(A_{1, n}+A_{3, n}\right)$, which follows from the proof of Theorem 2.1.

We remark that the upper bound in (2.29) of Corollary 2.4 is more efficient than the general upper bound obtained in (2.21) of Theorem 2.1 as the latter one converges to 0 more slowly.

Next, we apply Corollary 2.4 to some particular classes of covariance functions $r$.

Example 2.5 (Covariance Functions with Polynomial Decay). Assume that $d=1$ and $f=H_{q}$ with $q \geq 2$, and consider a covariance function $r$ which is regularly varying with parameter $a<0$. That is, for all $|k| \geq 1,|r(k)|=|k|^{a} l(|k|)$, where $l$ is a slowly varying function. Recall that for any regularly varying function $r$ with parameter $\alpha<0$, we have the following discrete version of Karamata's theorem (see e.g. [6]):

$$
\begin{array}{ll}
\alpha>-1: & \frac{\sum_{k=1}^{n}|r(k)|}{n^{a+1} l(n)} \rightarrow 1 /(\alpha+1), \\
\alpha<-1: & \frac{\sum_{k=n}^{\infty}|r(k)|}{n^{a+1} l(n)} \rightarrow-1 /(\alpha+1),
\end{array}
$$

as $n \rightarrow \infty$. Assume now that $a<-\frac{1}{q}$, which implies that the conditions of Theorem 2.1 are satisfied, and $a e \neq-1$ for any $e=1, \ldots, q-1$. By the aforementioned convergence results we immediately deduce the following estimates $(1 \leq e \leq q-1)$ :

$$
\begin{aligned}
& \sum_{|j| \leq n}|r(j)|^{q} \frac{|j|}{n} \lesssim n^{-1}+n^{a q+1} l(n), \\
& \sum_{|j|>n}|r(j)|^{q} \lesssim n^{a q+1} l(n), \\
& \gamma_{n, q, e} \lesssim n^{-1 / 2}+n^{a / 2} l(n)+n^{(a q+1) / 2} l^{2}(n) .
\end{aligned}
$$

Thus, for all three cases of Corollary 2.4 we conclude that

$$
\left|\mathbb{E}\left[h\left(S_{n}\right)\right]-\mathbb{E}[h(S)]\right| \lesssim \begin{cases}n^{-1 / 2}: & a<-1 \\ n^{a / 2} l(n): & a \in\left(-1,-\frac{1}{q-1}\right) \\ n^{\frac{a q+1}{2}} l^{2}(n): & a \in\left(-\frac{1}{q-1},-\frac{1}{q}\right) .\end{cases}
$$

Clearly, the same estimates hold for $d\left(S_{n}, S\right)$, where $d=d_{K o l}, d=d_{W}$ or $d=d_{C}$.

Example 2.6 (The Fractional Brownian Motion Case). Let $d=1, f=H_{q}$ with $q \geq 2$ and consider the fractional Gaussian noise $X_{i}=B_{i}^{H}-B_{i-1}^{H}$, where $B^{H}$ is a fractional Brownian 
motion with parameter $H \in(0,1)$. Recall that $B^{H}=\left(B_{t}^{H}\right)_{t \geq 0}$ is a centered Gaussian process (with stationary increments) with covariance structure given by

$$
\mathbb{E}\left[B_{t} B_{s}\right]=\frac{1}{2}\left(|t|^{2 H}+|s|^{2 H}-|t+s|^{2 H}\right) .
$$

It is well-known that the correlation function $r$ of the fractional Brownian noise has the following form:

$$
|r(k)|=|k|^{2 H-2} l(|k|), \quad k \geq 1,
$$

with $l(|k|) \rightarrow 2 H|2 H-1|$ as $|k| \rightarrow \infty$ when $H \neq \frac{1}{2}$, and $l(|k|)=0$ for $|k| \geq 1$ when $H=\frac{1}{2}$. As in the previous example we immediately deduce that

$$
\left|\mathbb{E}\left[h\left(S_{n}\right)\right]-\mathbb{E}[h(S)]\right| \lesssim \begin{cases}n^{-1 / 2}: & H \in\left(0, \frac{1}{2}\right] \\ n^{H-1 / 2}: & a \in\left[\frac{1}{2}, \frac{2 q-3}{2 q-2}\right] \\ n^{(2 q H-2 q+1) / 2}: & a \in\left[\frac{2 q-3}{2 q-2}, \frac{2 q-1}{2 q}\right)\end{cases}
$$

and the same estimates hold for $d\left(S_{n}, S\right)$ with $d=d_{K o l}, d=d_{W}$ or $d=d_{C}$. Let us remark that these upper bounds coincide with those derived in Theorem 4.1 in [25].

We finally remark that the rate $n^{-1 / 2}$ for $H \in\left(0, \frac{1}{2}\right]$ has been proved to be optimal in [26], i.e. $\left|\mathbb{E}\left[h\left(S_{n}\right)\right]-\mathbb{E}[h(S)]\right| \sim n^{-1 / 2}$. For the other two cases the optimality question is still an open problem.

\section{The toolbox}

\subsection{Malliavin calculus on a Gaussian space}

We shall now provide a short introduction to the tools of Malliavin calculus that are needed in the proof of our main Theorem 2.1. The reader is referred to [28] for any unexplained definition or result. Let $\mathfrak{H}$ be a real separable Hilbert space. We denote by $W=\{W(h): h \in \mathfrak{H}\}$ an isonormal Gaussian process over $\mathfrak{H}$, that is, $W$ is a centered Gaussian family indexed by the elements of $\mathfrak{H}$ and such that, for every $g_{1}, g_{2} \in \mathfrak{H}$,

$$
\mathbb{E}\left[W\left(g_{1}\right) W\left(g_{2}\right)\right]=\left\langle g_{1}, g_{2}\right\rangle_{\mathfrak{H}}
$$

In what follows, we shall use the notation $L^{2}(W)=L^{2}(\Omega, \sigma(W), \mathbb{P})$. For every $q \geq 1$, we write $\mathfrak{H}^{\otimes q}$ to indicate the $q$ th tensor power of $\mathfrak{H}$; the symbol $\mathfrak{H}^{\odot q}$ indicates the $q$ th symmetric tensor power of $\mathfrak{H}$, equipped with the norm $\sqrt{q !}\|\cdot\|_{\mathfrak{H}^{\otimes q}}$. We denote by $I_{q}$ the isometry between $\mathfrak{H}^{\odot q}$ and the $q$ th Wiener chaos of $X$. It is well-known (see again [28, Ch. 1]) that any random variable $F$ belonging to $L^{2}(W)$ admits the chaotic expansion:

$$
F=\sum_{q=0}^{\infty} I_{q}\left(f_{q}\right),
$$

where $I_{0}\left(f_{0}\right):=E[F]$, the series converges in $L^{2}$ and the kernels $f_{q} \in \mathfrak{H}^{\odot q}, q \geq 1$, are uniquely determined by $F$. In the particular case where $\mathfrak{H}=L^{2}(A, \mathscr{A}, \mu)$, with $(A, \mathscr{A})$ a measurable 
space and $\mu$ a $\sigma$-finite and non-atomic measure, one has that $\mathfrak{H}^{\odot q}=L_{s}^{2}\left(A^{q}, \mathscr{A}^{\otimes q}, \mu^{\otimes q}\right)$ is the space of symmetric and square integrable functions on $A^{q}$. Moreover, for every $f \in \mathfrak{H}^{\odot q}$, $I_{q}(f)$ coincides with the multiple Wiener-Itô integral (of order $q$ ) of $f$ with respect to $W$ (see [28, Ch. 1]). It is well-known that a random variable of the type $I_{q}(f), f \in \mathfrak{H}^{\odot q}$, has finite moments of all orders (see [18, Ch. VI]). For every $q \geq 0$, we write $J_{q}$ to indicate the orthogonal projection operator on the $q$ th Wiener chaos associated with $W$, so, if $F \in L^{2}(W)$ is as in (3.31), then $J_{q} F=I_{q}\left(f_{q}\right)$ for every $q \geq 0$.

Let $\left\{e_{k}, k \geq 1\right\}$ be a complete orthonormal system in $\mathfrak{H}$. Given $f \in \mathfrak{H}^{\odot p}$ and $g \in \mathfrak{H}^{\odot q}$, for every $r=0, \ldots, p \wedge q$, the $r$ th contraction of $f$ and $g$ is the element of $\mathfrak{H}^{\otimes(p+q-2 r)}$ defined as

$$
f \otimes_{r} g=\sum_{i_{1}, \ldots, i_{r}=1}^{\infty}\left\langle f, e_{i_{1}} \otimes \cdots \otimes e_{i_{r}}\right\rangle_{\mathfrak{H}^{\otimes r}} \otimes\left\langle g, e_{i_{1}} \otimes \cdots \otimes e_{i_{r}}\right\rangle_{\mathfrak{H}^{\otimes r}}
$$

In the particular case where $\mathfrak{H}=L^{2}(A, \mathscr{A}, \mu)$ (with $\mu$ non-atomic), one has that

$$
\begin{aligned}
f \otimes_{r} g= & \int_{A^{r}} f\left(t_{1}, \ldots, t_{p-r}, s_{1}, \ldots, s_{r}\right) \\
& \times g\left(t_{p-r+1}, \ldots, t_{p+q-2 r}, s_{1}, \ldots, s_{r}\right) d \mu\left(s_{1}\right) \ldots d \mu\left(s_{r}\right) .
\end{aligned}
$$

Moreover, $f \otimes_{0} g=f \otimes g$ equals the tensor product of $f$ and $g$ while, for $p=q$, $f \otimes_{p} g=\langle f, g\rangle_{\mathfrak{H}} \otimes p$. Note that, in general, the contraction $f \otimes_{r} g$ is not a symmetric element of $\mathfrak{H}^{\otimes(p+q-2 r)}$. The canonical symmetrization of $f \otimes_{r} g$ is written as $f \widetilde{\otimes}_{r} g$. The following multiplication formula is also very useful: if $f \in \mathfrak{H}^{\odot p}$ and $g \in \mathfrak{H}^{\odot q}$, then

$$
I_{p}(f) I_{q}(g)=\sum_{r=0}^{p \wedge q} r !\left(\begin{array}{l}
p \\
r
\end{array}\right)\left(\begin{array}{l}
q \\
r
\end{array}\right) I_{p+q-2 r}\left(f \widetilde{\otimes}_{r} g\right) .
$$

Let $\mathscr{S}$ be the set of all smooth cylindrical random variables of the form

$$
F=g\left(W\left(\phi_{1}\right), \ldots, W\left(\phi_{n}\right)\right),
$$

where $n \geq 1, g: \mathbb{R}^{n} \rightarrow \mathbb{R}$ is a smooth function with compact support and $\phi_{i} \in \mathfrak{H}$. The Malliavin derivative of $F$ with respect to $W$ is the element of $L^{2}(\Omega, \mathfrak{H})$ defined as

$$
D F=\sum_{i=1}^{n} \frac{\partial g}{\partial x_{i}}\left(W\left(\phi_{1}\right), \ldots, W\left(\phi_{n}\right)\right) \phi_{i}
$$

Also, $D W(\phi)=\phi$ for every $\phi \in \mathfrak{H}$. As usual, $\mathbb{D}^{1,2}$ denotes the closure of $\mathscr{S}$ with respect to the norm $\|\cdot\|_{1,2}$, defined by the relation

$$
\|F\|_{1,2}^{2}=\mathbb{E}\left[F^{2}\right]+\mathbb{E}\left[\|D F\|_{\mathfrak{H}}^{2}\right] .
$$

Note that, if $F$ is equal to a finite sum of multiple Wiener-Itô integrals, then $F \in \mathbb{D}^{1,2}$. The Malliavin derivative $D$ verifies the following chain rule: if $\varphi: \mathbb{R}^{n} \rightarrow \mathbb{R}$ is in $\mathscr{C}_{b}^{1}$ (that is, the collection of continuously differentiable functions with bounded partial derivatives) and if $\left\{F_{i}\right\}_{i=1, \ldots, n}$ is a vector of elements of $\mathbb{D}^{1,2}$, then $\varphi\left(F_{1}, \ldots, F_{n}\right) \in \mathbb{D}^{1,2}$ and 


$$
D \varphi\left(F_{1}, \ldots, F_{n}\right)=\sum_{i=1}^{n} \frac{\partial \varphi}{\partial x_{i}}\left(F_{1}, \ldots, F_{n}\right) D F_{i} .
$$

We denote by $\delta$ the adjoint of the operator $D$, also called the divergence operator. A random element $u \in L^{2}(\Omega, \mathfrak{H})$ belongs to the domain of $\delta$, denoted as Dom $\delta$, if and only if it verifies

$$
\left|\mathbb{E}\langle D F, u\rangle_{\mathfrak{H}}\right| \leq c_{u}\|F\|_{L^{2}} \text { for any } F \in \mathscr{S},
$$

where $c_{u}$ is a constant depending only on $u$. If $u \in \operatorname{Dom} \delta$, then the random variable $\delta(u)$ is defined by the duality relationship (sometimes called the 'integration by parts formula')

$$
\mathbb{E}[F \delta(u)]=\mathbb{E}\langle D F, u\rangle_{\mathfrak{H}},
$$

which holds for every $F \in \mathbb{D}^{1,2}$.

The operator $L$, acting on square integrable random variables of the type (3.31), is defined through the projection operators $\left\{J_{q}\right\}_{q \geq 0}$ as $L=\sum_{q=0}^{\infty}-q J_{q}$, and is called the infinitesimal generator of the Ornstein-Uhlenbeck semigroup. It verifies the following crucial property: a random variable $F$ is an element of $\operatorname{Dom} L\left(=\mathbb{D}^{2,2}\right)$ if, and only if, $F \in \operatorname{Dom} \delta D$ (i.e. $F \in \mathbb{D}^{1,2}$ and $D F \in \operatorname{Dom} \delta$ ), and in this case, $\delta D F=-L F$. Note that a random variable $F$ as in (3.31) is in $\mathbb{D}^{1,2}$ if and only if

$$
\sum_{q=1}^{\infty}(q+1) !\left\|f_{q}\right\|_{\mathfrak{H}^{\otimes q}}^{2}<\infty
$$

and also $\mathbb{E}\left[\|D F\|_{\mathfrak{H}}^{2}\right]=\sum_{q \geq 1} q q !\left\|f_{q}\right\|_{\mathfrak{H} \otimes q}^{2}$. If $\mathfrak{H}=L^{2}(A, \mathscr{A}, \mu)$ (with $\mu$ non-atomic), then the derivative of a random variable $F$ as in (3.31) can be identified with the element of $L^{2}(A \times \Omega)$ given by

$$
D_{a} F=\sum_{q=1}^{\infty} q I_{q-1}\left(f_{q}(\cdot, a)\right), \quad a \in A
$$

We also define the operator $L^{-1}$, which is the pseudo-inverse of $L$, as follows: for every $F \in L^{2}(W)$, we set $L^{-1} F=\sum_{q \geq 1} \frac{1}{q} J_{q}(F)$. Note that $L^{-1}$ is an operator with values in $\mathbb{D}^{2,2}$ and that $L L^{-1} F=F-\mathbb{E}[F]$ for all $F \in L^{2}(W)$.

\subsection{Assessing norms and scalar products}

The following statement plays a crucial role in the proof of Theorem 2.1.

Lemma 3.1. Let $F=I_{p}(h)$ and $G=I_{s}(g)$ with $h \in \mathfrak{H}^{\odot p}, g \in \mathfrak{H}^{\odot s}$ and $p<s(p, s \geq 1)$. Then

$$
\operatorname{Var}\left[\frac{1}{s}\|D G\|_{\mathfrak{H}}^{2}\right]=\frac{1}{s^{2}} \sum_{l=1}^{s-1} l^{2} l !^{2}\left(\begin{array}{l}
s \\
l
\end{array}\right)^{4}(2 s-2 l) !\left\|g \widetilde{\otimes}_{l} g\right\|_{\mathfrak{H}^{\otimes 2 s-2 l}}^{2},
$$

and

$$
\mathbb{E}\left[\left(\frac{1}{s}\langle D F, D G\rangle_{\mathfrak{H}}\right)^{2}\right] \leq p !\left(\begin{array}{c}
s-1 \\
p-1
\end{array}\right)^{2}(s-p) ! \mathbb{E}\left[F^{2}\right]\left\|g \otimes_{s-p} g\right\|_{\mathfrak{H}^{\otimes 2 p}}
$$




$$
\begin{aligned}
& +\frac{p^{2}}{2} \sum_{l=1}^{p-1}(l-1) !^{2}\left(\begin{array}{c}
p-1 \\
l-1
\end{array}\right)^{2}\left(\begin{array}{c}
s-1 \\
l-1
\end{array}\right)^{2}(p+s-2 l) ! \\
& \times\left(\left\|h \otimes_{p-l} h\right\|_{\mathfrak{H}^{\otimes 2 l}}^{2}+\left\|g \otimes_{s-l} g\right\|_{\mathfrak{H}^{\otimes 2 l}}^{2}\right) .
\end{aligned}
$$

Proof of 3.36. We have $D G=s I_{s-1}(g)$, and so, by using (3.33),

$$
\begin{aligned}
\frac{1}{s}\|D G\|_{\mathfrak{H}}^{2} & =s\left\|I_{s-1}(g)\right\|_{\mathfrak{H}}^{2}=s \sum_{l=0}^{s-1} l !\left(\begin{array}{c}
s-1 \\
l
\end{array}\right)^{2} I_{2 s-2-2 l}\left(g \widetilde{\otimes}_{l+1} g\right) \\
& =s \sum_{l=1}^{s}(l-1) !\left(\begin{array}{c}
s-1 \\
l-1
\end{array}\right)^{2} I_{2 s-2 l}\left(g \widetilde{\otimes}_{l} g\right) \\
& =s !\|g\|_{\mathfrak{H}^{\otimes s}}^{2}+s \sum_{l=1}^{s-1}(l-1) !\left(\begin{array}{c}
s-1 \\
l-1
\end{array}\right)^{2} I_{2 s-2 l}\left(g \widetilde{\otimes}_{l} g\right) \\
& =\mathbb{E}\left[G^{2}\right]+s \sum_{l=1}^{s-1}(l-1) !\left(\begin{array}{c}
s-1 \\
l-1
\end{array}\right)^{2} I_{2 s-2 l}\left(g \widetilde{\otimes}_{l} g\right) .
\end{aligned}
$$

The orthogonality property of multiple integrals leads to (3.36).

Proof of 3.37. Thanks once again to (3.33), we can write

$$
\begin{aligned}
\langle D F, D G\rangle_{\mathfrak{H}} & =p s\left\langle I_{p-1}(h), I_{s-1}(g)\right\rangle_{\mathfrak{H}} \\
& =p s \sum_{l=0}^{p \wedge s-1} l !\left(\begin{array}{c}
p-1 \\
l
\end{array}\right)\left(\begin{array}{c}
s-1 \\
l
\end{array}\right) I_{p+s-2-2 l}\left(h \widetilde{\otimes}_{l+1} g\right) \\
& =p s \sum_{l=1}^{p \wedge s}(l-1) !\left(\begin{array}{c}
p-1 \\
l-1
\end{array}\right)\left(\begin{array}{c}
s-1 \\
l-1
\end{array}\right) I_{p+s-2 l}\left(h \widetilde{\otimes}_{l} g\right) .
\end{aligned}
$$

It follows that

$$
\begin{aligned}
\mathbb{E}[( & \left.\left.\frac{1}{s}\langle D F, D G\rangle_{\mathfrak{H}}\right)^{2}\right]=p^{2} \sum_{l=1}^{p}(l-1) !^{2} \\
& \times\left(\begin{array}{c}
p-1 \\
l-1
\end{array}\right)^{2}\left(\begin{array}{c}
s-1 \\
l-1
\end{array}\right)^{2}(p+s-2 l) !\left\|h \widetilde{\otimes}_{l} g\right\|_{\mathfrak{H}^{\otimes(p+s-2 l)}}^{2} .
\end{aligned}
$$

If $l<p$, then

$$
\begin{aligned}
\left\|h \widetilde{\otimes}_{l} g\right\|_{\mathfrak{H}^{\otimes(p+s-2 l)}}^{2} & \leq\left\|h \otimes_{l} g\right\|_{\mathfrak{H}^{\otimes(p+s-2 l)}}^{2}=\left\langle h \otimes_{p-l} h, g \otimes_{s-l} g\right\rangle_{\mathfrak{H}^{\otimes 2 l}} \\
& \leq\left\|h \otimes_{p-l} h\right\|_{\mathfrak{H}^{\otimes 2 l}}\left\|g \otimes_{s-l} g\right\|_{\mathfrak{H}^{\otimes 2 l}} \\
& \leq \frac{1}{2}\left(\left\|h \otimes_{p-l} h\right\|_{\mathfrak{H}^{\otimes 2 l}}^{2}+\left\|g \otimes_{s-l} g\right\|_{\mathfrak{H}^{\otimes 2 l}}^{2}\right) .
\end{aligned}
$$

If $l=p$, then

$$
\left\|h \widetilde{\otimes}_{p} g\right\|_{\mathfrak{H}^{\otimes(s-p)}}^{2} \leq\left\|h \otimes_{p} g\right\|_{\mathfrak{H}^{\otimes(s-p)}}^{2} \leq\|h\|_{\mathfrak{H}^{\otimes p}}^{2}\left\|g \otimes_{s-p} g\right\|_{\mathfrak{H}^{\otimes 2 p}} .
$$

By plugging these last expressions into (3.38), we deduce immediately (3.37). 


\subsection{Estimates via interpolations and Stein's method}

The forthcoming Theorem 3.2 contains two bounds on normal approximations, that are expressed in terms of Malliavin operators. As anticipated, the proof of Point (1) uses an interpolation technique already applied in [27,30], which is close to the 'smart path method' of spin glasses [35]. Point (2) uses estimates from [25].

Theorem 3.2. Let $F$ be a centered element of $\mathbb{D}^{1,2}$ and let $Z \sim N\left(0, \sigma^{2}\right), \sigma>0$.

(1) Suppose that $h: \mathbb{R} \rightarrow \mathbb{R}$ is twice continuously differentiable and has a bounded second derivative. Then,

$$
|\mathbb{E}[h(F)]-\mathbb{E}[h(Z)]| \leq \frac{\left\|h^{\prime \prime}\right\|_{\infty}}{2} \mathbb{E}\left|\sigma^{2}-\left\langle D F,-D L^{-1} F\right\rangle_{\mathfrak{H}}\right| .
$$

(2) Suppose that $h: \mathbb{R} \rightarrow \mathbb{R}$ is Lipschitz. Then,

$$
|\mathbb{E}[h(F)]-\mathbb{E}[h(Z)]| \leq \frac{\left\|h^{\prime}\right\|_{\infty}}{\sigma} \mathbb{E}\left|\sigma^{2}-\left\langle D F,-D L^{-1} F\right\rangle_{\mathfrak{H}}\right| .
$$

Proof. (1) Without loss of generality, we may assume that $F$ and $Z$ are independent and defined on the same probability space. Fix $h$ as in the statement, and define the function $\Psi(t)=\mathbb{E}[h(\sqrt{1-t} F+\sqrt{t} Z)], t \in[0,1]$. Standard results imply that $\Psi$ is differentiable for every $t \in(0,1)$, and that

$$
\Psi^{\prime}(t)=\frac{1}{2 \sqrt{t}} \mathbb{E}\left[h^{\prime}(\sqrt{1-t} F+\sqrt{t} Z) Z\right]-\frac{1}{2 \sqrt{1-t}} \mathbb{E}\left[h^{\prime}(\sqrt{1-t} F+\sqrt{t} Z) F\right] .
$$

By using independence and integration by parts, we obtain immediately that

$$
\frac{1}{2 \sqrt{t}} \mathbb{E}\left[h^{\prime}(\sqrt{1-t} F+\sqrt{t} Z) Z\right]=\frac{\sigma^{2}}{2} \mathbb{E}\left[h^{\prime \prime}(\sqrt{1-t} F+\sqrt{t} Z)\right] \text {. }
$$

On the other hand, the relation $F=L L^{-1} F=-\delta D L^{-1} F$ and (3.34) imply that

$$
\begin{aligned}
& \frac{1}{2 \sqrt{1-t}} \mathbb{E}\left[h^{\prime}(\sqrt{1-t} F+\sqrt{t} S) F\right] \\
& =\frac{1}{2 \sqrt{1-t}} \mathbb{E}\left[h^{\prime}(\sqrt{1-t} F+\sqrt{t} Z) \delta\left(-D L^{-1} F\right)\right] \\
& =\frac{1}{2} \mathbb{E}\left[h^{\prime \prime}(\sqrt{1-t} F+\sqrt{t} Z)\left\langle D F,-D L^{-1} F\right\rangle_{\mathfrak{H}}\right] .
\end{aligned}
$$

The conclusion follows from the fact that

$$
|\mathbb{E}[h(F)]-\mathbb{E}[h(Z)]|=|\Psi(1)-\Psi(0)| \leq \int_{0}^{1}\left|\Psi^{\prime}(t)\right| d t .
$$

(2) Here we follow the arguments contained in the proof of Theorem 3.1 in [25]. Define $h_{\sigma}(x)=h(\sigma x), F_{\sigma}=\sigma^{-1} F$, and $Z_{\sigma}=\sigma^{-1} Z \sim N(0,1)$. Let $s$ be the solution of the Stein equation associated with $h_{\sigma}$, i.e. $s$ solves the differential equation

$$
h_{\sigma}(x)-\mathbb{E}\left[h_{\sigma}\left(Z_{\sigma}\right)\right]=s^{\prime}(x)-x s(x), \quad x \in \mathbb{R} .
$$

It is well-known that such a solution is given by $s(x)=\frac{1}{\varphi(x)} \int_{-\infty}^{x}\left(h_{\sigma}(t)-\Phi(t)\right) \varphi(t) d t$, where $\varphi$ and $\Phi$ are the density and the distribution function of $N(0,1)$, respectively, and $\left\|s^{\prime}\right\|_{\infty} \leq\left\|h_{\sigma}^{\prime}\right\|_{\infty}$. 
Since $F_{\sigma}$ is a centered element of $\mathbb{D}^{1,2}$ it holds that $F_{\sigma}=L L^{-1} F_{\sigma}=-\delta D L^{-1} F_{\sigma}$. By the integration by parts formula (3.34) we deduce that

$$
\begin{aligned}
|\mathbb{E}[h(F)]-\mathbb{E}[h(Z)]| & =\left|\mathbb{E}\left[h_{\sigma}\left(F_{\sigma}\right)\right]-\mathbb{E}\left[h_{\sigma}\left(Z_{\sigma}\right)\right]\right| \\
& =\left|\mathbb{E}\left[s^{\prime}\left(F_{\sigma}\right)-F_{\sigma} s\left(F_{\sigma}\right)\right]\right| \\
& =\left|\mathbb{E}\left[s^{\prime}\left(F_{\sigma}\right)\left(1-\left\langle D F_{\sigma},-D L^{-1} F_{\sigma}\right\rangle_{\mathfrak{H}}\right)\right]\right| \\
& \leq\left\|s^{\prime}\right\|_{\infty} \mathbb{E}\left|1-\left\langle D F_{\sigma},-D L^{-1} F_{\sigma}\right\rangle_{\mathfrak{H}}\right| \\
& \leq\left\|h_{\sigma}^{\prime}\right\|_{\infty} \mathbb{E}\left|1-\left\langle D F_{\sigma},-D L^{-1} F_{\sigma}\right\rangle_{\mathfrak{H}}\right| .
\end{aligned}
$$

We conclude by using the relations $\left\|h_{\sigma}^{\prime}\right\|_{\infty}=\sigma\left\|h^{\prime}\right\|_{\infty}$ and

$$
\left\langle D F_{\sigma},-D L^{-1} F_{\sigma}\right\rangle_{\mathfrak{H}}=\sigma^{-2}\left\langle D F,-D L^{-1} F\right\rangle_{\mathfrak{H}}
$$

When applied to the special case of a Gaussian random variable $F$, Theorem 3.2 yields the following neat estimates.

Corollary 3.3. Let $F \sim N\left(0, \gamma^{2}\right)$ and $Z \sim N\left(0, \sigma^{2}\right), \gamma, \sigma>0$.

(1) For every $h$ twice continuously differentiable and with a bounded second derivative,

$$
|\mathbb{E}[h(F)]-\mathbb{E}[h(Z)]| \leq \frac{\left\|h^{\prime \prime}\right\|_{\infty}}{2}\left|\sigma^{2}-\gamma^{2}\right| .
$$

(2) For every Lipschitz function $h$,

$$
|\mathbb{E}[h(F)]-\mathbb{E}[h(Z)]| \leq \frac{\left\|h^{\prime}\right\|_{\infty}}{\sigma \vee \gamma}\left|\sigma^{2}-\gamma^{2}\right| .
$$

\section{Proof of Theorem 2.1}

\subsection{Preparation}

First, let us remark that the process $X=\left(X_{k}\right)_{k \in \mathbb{Z}}$ can always be regarded as a subset of an isonormal Gaussian process $\{W(u): u \in \mathfrak{H}\}$, where $\mathfrak{H}$ is a separable Hilbert space with scalar product $\langle\cdot, \cdot\rangle_{\mathfrak{H}}$. More precisely, we shall assume (without loss of generality) that, for every $k \in \mathbb{Z}$ and every $1 \leq l \leq d$, there exists $u_{k, l} \in \mathfrak{H}$ such that

$$
X_{k}^{(l)}=W\left(u_{k, l}\right), \quad \text { and consequently }\left\langle u_{k, l}, u_{k^{\prime}, l^{\prime}}\right\rangle_{\mathfrak{H}}=r^{\left(l, l^{\prime}\right)}\left(k-k^{\prime}\right),
$$

for every $k, k^{\prime} \in \mathbb{Z}$ and every $1 \leq l, l^{\prime} \leq d$. Observe also that $\mathfrak{H}$ can be taken of the form $\mathfrak{H}=L^{2}(A, \mathscr{A}, \mu)$, where $\mu$ is $\sigma$-finite and non-atomic.

Using the Hermite expansion (2.6) of the function $f$ we obtain the Wiener chaos representation

$$
S_{n}=\sum_{m=q}^{\infty} I_{m}\left(g_{m}^{n}\right), \quad g_{m}^{n} \in \mathfrak{H}^{\odot m},
$$

where the kernels $g_{m}^{n}$ have the form

$$
g_{m}^{n}=\frac{1}{\sqrt{n}} \sum_{k=1}^{n} \sum_{t \in\{1, \ldots, d\}^{m}} b_{t} u_{k, t_{1}} \otimes \cdots \otimes u_{k, t_{m}}
$$


for certain coefficients $b_{t}$ such that the mapping $t \mapsto b_{t}$ is symmetric on $\{1, \ldots, d\}^{m}$. One also has the identities

$$
\mathbb{E}\left[f_{m}^{2}\left(X_{1}\right)\right]=m ! \sum_{t \in\{1, \ldots, d\}^{m}} b_{t}^{2}, \quad m \geq q, \quad \mathbb{E}\left[f^{2}\left(X_{1}\right)\right]=\sum_{m=q}^{\infty} m ! \sum_{t \in\{1, \ldots, d\}^{m}} b_{t}^{2} .
$$

Here is a useful preliminary result.

Lemma 4.1. For the kernels $g_{m}^{n}$ defined in (4.43) and any $1 \leq e \leq m-1$ we obtain the inequality, valid for every $n$,

$$
\left\|g_{m}^{n} \otimes_{e} g_{m}^{n}\right\|_{\mathfrak{H}^{\otimes 2(m-e)}} \leq \frac{d^{m}}{m !} \mathbb{E}\left[f_{m}^{2}\left(X_{1}\right)\right] \gamma_{n, m, e},
$$

where $\gamma_{n, m, e}$ is defined by (2.12). Furthermore, we have that, for every $n$,

$$
m !\left\|g_{m}^{n}\right\|_{\mathfrak{H}^{\otimes m}}^{2} \leq \mathbb{E}\left[f_{m}^{2}\left(X_{1}\right)\right]\left(2 K+d^{q} \theta\right),
$$

where the constants $K$ and $\theta$ are defined, respectively, in (2.9) and (2.10).

Proof of 4.44. Fix $1 \leq e \leq m-1$. Observe that

$$
\begin{aligned}
g_{m}^{n} \otimes_{e} g_{m}^{n}= & \frac{1}{n} \sum_{k_{1}, k_{2}=1}^{n} \sum_{t, s \in\{1, \ldots, d\}^{m}} b_{t} b_{s} \prod_{j=1}^{e} r^{\left(t_{j}, s_{j}\right)}\left(k_{1}-k_{2}\right) \\
& \times u_{k_{1}, t_{p+1}} \otimes \cdots \otimes u_{k_{1}, t_{m}} \otimes u_{k_{2}, s_{p+1}} \otimes \cdots \otimes u_{k_{2}, s_{m}} .
\end{aligned}
$$

We obtain

$$
\begin{aligned}
\| g_{m}^{n} & \otimes_{e} g_{m}^{n} \|_{\mathfrak{H}^{\otimes 2(m-e)}}^{2} \leq\left(\frac{d^{m}}{m !} \mathbb{E}\left[f_{m}^{2}\left(X_{1}\right)\right]\right)^{2} \\
& \times n^{-2} \sum_{k_{1}, \ldots, k_{4}=1}^{n} \theta\left(k_{1}-k_{2}\right)^{e} \theta\left(k_{3}-k_{4}\right)^{e} \theta\left(k_{1}-k_{3}\right)^{m-e} \theta\left(k_{2}-k_{4}\right)^{m-e},
\end{aligned}
$$

where $\theta(j)$ is defined in (2.8). Since $\theta\left(k_{3}-k_{4}\right)^{e} \theta\left(k_{1}-k_{3}\right)^{m-e} \leq \theta\left(k_{3}-k_{4}\right)^{m}+\theta\left(k_{1}-k_{3}\right)^{m}$ we deduce that

$$
\begin{aligned}
& n^{-2} \sum_{k_{1}, \ldots, k_{4}=1}^{n} \theta\left(k_{1}-k_{2}\right)^{e} \theta\left(k_{3}-k_{4}\right)^{e} \theta\left(k_{1}-k_{3}\right)^{m-e} \theta\left(k_{2}-k_{4}\right)^{m-e} \\
& \leq 2 n^{-1} \sum_{k \in \mathbb{Z}} \theta(k)^{m} \sum_{|k| \leq n} \theta(k)^{e} \sum_{|k| \leq n} \theta(k)^{m-e} \leq \gamma_{n, m, e}^{2} .
\end{aligned}
$$

Hence, we obtain (4.44).

Proof of 4.45. By the Cauchy-Schwarz inequality we have

$$
\begin{aligned}
& m ! \mid\left\langle\sum_{t \in\{1, \ldots, d\}^{m}} b_{t} u_{k, t_{1}} \otimes \cdots \otimes u_{k, t_{m}},\left.\sum_{t \in\{1, \ldots, d\}^{m}} b_{t} u_{k+l, t_{1}} \otimes \cdots \otimes u_{k+l, t_{m}}\right|_{\mathfrak{H}^{\otimes m}}\right| \\
& \quad \leq \mathbb{E}\left[f_{m}^{2}\left(X_{1}\right)\right] .
\end{aligned}
$$


We deduce, for any $m \geq q$,

$$
\begin{aligned}
m !\left\|g_{m}^{n}\right\|_{\mathfrak{H}^{\otimes m}}^{2} & =\frac{m !}{n} \sum_{k_{1}, k_{2}=1}^{n} \sum_{t, s \in\{1, \ldots, d\}^{m}} b_{t} b_{s} \prod_{j=1}^{m} r^{\left(t_{j}, s_{j}\right)}\left(k_{1}-k_{2}\right) \\
& \leq\left(2 H \mathbb{E}\left[f_{m}^{2}\left(X_{1}\right)\right]+m ! \sum_{|k| \geq K} \theta(k)^{m}\left(\sum_{t \in\{1, \ldots, d\}^{m}}\left|b_{t}\right|\right)^{2}\right) \\
& \leq \mathbb{E}\left[f_{m}^{2}\left(X_{1}\right)\right]\left(2 K+\sum_{|k| \geq K}(d \theta(k))^{m}\right) \leq \mathbb{E}\left[f_{m}^{2}\left(X_{1}\right)\right]\left(2 K+d^{q} \theta\right),
\end{aligned}
$$

which implies (4.45).

The proofs of Point 1 and Point 2 in Theorem 2.1 are similar, and are detailed in the subsequent two sections.

\subsection{Proof of Theorem 2.1-(1)}

First of all, we remark that $\theta(j) \rightarrow 0$ as $|j| \rightarrow \infty$, because $\sum_{j \in \mathbb{Z}} \theta(j)^{q}<\infty$. This implies that $K<\infty$, where the constant $K$ is defined in (2.9). Moreover, the asymptotic variance $\sigma^{2}$ is finite. Indeed we have that $\sigma^{2}=\sum_{m=q}^{\infty} m !\left\|g_{m}^{n}\right\|_{\mathfrak{H}^{\otimes m}}^{2} \leq \mathbb{E}\left[f^{2}\left(X_{1}\right)\right]\left(2 K+d^{q} \theta\right)$ due to (4.45).

The main proof is composed of several steps.

(a) Reduction to a finite chaos expansion. We start by approximating $S_{n}$ by a finite sum of multiple integrals. Define

$$
S_{n, N}=\sum_{m=q}^{N} I_{m}\left(g_{m}^{n}\right) .
$$

Now, let $h \in C^{2}(\mathbb{R})$ be a function with bounded second derivative. Since

$$
\left|h(x)-h(y)-h^{\prime}(0)(x-y)\right| \leq \frac{1}{2}\left\|h^{\prime \prime}\right\|_{\infty}(y-x)^{2}+\left\|h^{\prime \prime}\right\|_{\infty}|x||y-x|
$$

for all $x, y \in \mathbb{R}$, we immediately obtain that

$$
\begin{aligned}
& \left|\mathbb{E}\left[h\left(S_{n}\right)\right]-\mathbb{E}\left[h\left(S_{n, N}\right)\right]\right| \leq\left\|h^{\prime \prime}\right\|_{\infty} \\
& \quad \times\left(\frac{1}{2}\left\|S_{n}-S_{n, N}\right\|_{L^{2}(\mathbb{P})}^{2}+\left\|S_{n}\right\|_{L^{2}(\mathbb{P})}\left\|S_{n}-S_{n, N}\right\|_{L^{2}(\mathbb{P})}\right) .
\end{aligned}
$$

By inequality (4.45) we deduce that

$$
\left\|S_{n}\right\|_{L^{2}(\mathbb{P})}^{2} \leq\left(2 K+d^{q} \theta\right)\left\|f\left(X_{1}\right)\right\|_{L^{2}(\mathbb{P})}^{2}
$$

and

$$
\begin{aligned}
\left\|S_{n}-S_{n, N}\right\|_{L^{2}(\mathbb{P})}^{2} & \leq\left(2 K+d^{q} \theta\right) \sum_{m=N+1}^{\infty}\left\|f_{m}\left(X_{1}\right)\right\|_{L^{2}(\mathbb{P})}^{2} \\
& \leq\left(2 K+d^{q} \theta\right)\left\|f\left(X_{1}\right)\right\|_{L^{2}(\mathbb{P})}\left(\sum_{m=N+1}^{\infty}\left\|f_{m}\left(X_{1}\right)\right\|_{L^{2}(\mathbb{P})}^{2}\right)^{1 / 2},
\end{aligned}
$$


where $\theta$ is defined by (2.10). We conclude that

$$
\begin{aligned}
\left|\mathbb{E}\left[h\left(S_{n}\right)\right]-\mathbb{E}\left[h\left(S_{n, N}\right)\right]\right| \leq & \frac{3\left(2 K+d^{q} \theta\right)}{2}\left\|h^{\prime \prime}\right\|_{\infty}\left\|f\left(X_{1}\right)\right\|_{L^{2}(\mathbb{P})} \\
& \times\left(\sum_{m=N+1}^{\infty}\left\|f_{m}\left(X_{1}\right)\right\|_{L^{2}(\mathbb{P})}^{2}\right)^{1 / 2},
\end{aligned}
$$

which completes the first step.

(b) Bounds based on the interpolation inequality (3.39). Let $Z_{N}$ be a centered Gaussian random variable with variance $\sum_{m=q}^{N} \sigma_{m}^{2}$. By using (3.39) in the special case $F=S_{n, N}, Z=Z_{N}$ and by applying e.g. (3.35), we obtain

$$
\begin{aligned}
& \mathbb{E}\left[\left[h\left(Z_{N}\right)\right]-\mathbb{E}\left[h\left(S_{n, N}\right)\right]\right] \leq \frac{1}{2}\left\|h^{\prime \prime}\right\|_{\infty}\left\|\sum_{m=q}^{N} \sigma_{m}^{2}-\left\langle D S_{n, N},-D L^{-1} S_{n, N}\right\rangle_{\mathfrak{H}}\right\|_{L^{2}(\mathbb{P})} \\
& \quad \leq \frac{1}{2}\left\|h^{\prime \prime}\right\|_{\infty} \sum_{p, s=q}^{N}\left\|\delta_{p s} \sigma_{p}^{2}-s^{-1}\left\langle D I_{p}\left(g_{p}^{n}\right), D I_{s}\left(g_{s}^{n}\right)\right\rangle_{\mathfrak{H}}\right\|_{L^{2}(\mathbb{P})},
\end{aligned}
$$

where $\delta_{p s}$ is the Kronecker symbol. This completes the second step.

(c) The final estimates. Here we give the approximation of the term on the right-hand side of (4.47). By (4.45) and the dominating convergence theorem we immediately deduce that

$$
\mathbb{E}\left[s^{-1}\left\|D I_{s}\left(g_{s}^{n}\right)\right\|_{\mathfrak{H}}^{2}\right]=s !\left\|g_{s}^{n}\right\|_{\mathfrak{H}^{\otimes s}}^{2} \rightarrow \sigma_{s}^{2}=s ! \sum_{k \in \mathbb{Z}} \sum_{t, l \in\{1, \ldots, d\}^{s}} b_{t} b_{l} \prod_{j=1}^{m} r^{\left(t_{j}, l_{j}\right)}(k) .
$$

As in the proof of (4.45) we conclude that (recall that we assumed $n>K$ )

$$
\begin{aligned}
\left|\mathbb{E}\left[s^{-1}\left\|D I_{s}\left(g_{s}^{n}\right)\right\|_{\mathfrak{H}}^{2}\right]-\sigma_{s}^{2}\right| \leq & s ! \sum_{|k|<K}\left|\sum_{t, l \in\{1, \ldots, d\}^{s}} b_{t} b_{l} \prod_{j=1}^{m} r^{\left(t_{j}, l_{j}\right)}(k)\right| \frac{|k|}{K} \\
& +s ! \sum_{K \leq|k|<n}\left|\sum_{t, l \in\{1, \ldots, d\}^{s}} b_{t} b_{l} \prod_{j=1}^{m} r^{\left(t_{j}, l_{j}\right)}(k)\right| \frac{|k|}{K} \\
& +s ! \sum_{|k| \geq n}\left|\sum_{t, l \in\{1, \ldots, d\}^{s}} b_{t} b_{l} \prod_{j=1}^{m} r^{\left(t_{j}, l_{j}\right)}(k)\right| \\
\leq & \mathbb{E}\left[f_{s}^{2}\left(X_{1}\right)\right]\left\{\frac{2 K^{2}}{n}+d^{q}\left(\sum_{|j| \leq n} \theta(j)^{q} \frac{|j|}{n}+\sum_{|j|>n} \theta(j)^{q}\right)\right\} .
\end{aligned}
$$

Thus we have

$$
\begin{aligned}
& \sum_{s=q}^{N}\left\|\sigma_{s}^{2}-s^{-1}\right\| D I_{s}\left(g_{s}^{n}\right)\left\|_{\mathfrak{H}}^{2}\right\|_{L^{2}(\mathbb{P})} \\
& \quad \leq \sum_{s=q}^{N}\left(\left|\mathbb{E}\left[s^{-1}\left\|D I_{S}\left(g_{s}^{n}\right)\right\|_{\mathfrak{H}}^{2}\right]-\sigma_{s}^{2}\right|+\sqrt{\operatorname{Var}\left[s^{-1}\left\|D I_{s}\left(g_{s}^{n}\right)\right\|_{\mathfrak{H}}^{2}\right]}\right)
\end{aligned}
$$




$$
\begin{aligned}
\leq & \mathbb{E}\left[f_{s}^{2}\left(X_{1}\right)\right]\left\{\frac{2 H^{2}}{n}+d^{q}\left(\sum_{|j| \leq n} \theta(j)^{q} \frac{|j|}{n}+\sum_{|j|>n} \theta(j)^{q}\right)\right\} \\
& +\sum_{s=q}^{N} \sqrt{\operatorname{Var}\left[s^{-1}\left\|D I_{s}\left(g_{s}^{n}\right)\right\|_{\mathfrak{H}}^{2}\right]} \\
\leq & 2\left(A_{1, n}+A_{3, n, N}\right),
\end{aligned}
$$

where the last inequality follows directly from Lemmas 3.1 and 4.1. On the other hand, using the approximation (4.45) and Lemmas 3.1 and 4.1 once again we deduce that

$$
\begin{aligned}
& \sum_{q \leq p<s \leq N}\left\|s^{-1}\left\langle D I_{p}\left(g_{p}^{n}\right), D I_{s}\left(g_{s}^{n}\right)\right\rangle_{\mathfrak{H}}\right\|_{L^{2}(\mathbb{P})}+\sum_{q \leq s<p \leq N}\left\|s^{-1}\left\langle D I_{p}\left(g_{p}^{n}\right), D I_{s}\left(g_{s}^{n}\right)\right\rangle_{\mathfrak{H}}\right\|_{L^{2}(\mathbb{P})} \\
= & \sum_{q \leq p<s \leq N} \frac{p+s}{p}\left\|s^{-1}\left\langle D I_{p}\left(g_{p}^{n}\right), D I_{s}\left(g_{s}^{n}\right)\right\rangle_{\mathfrak{H}}\right\|_{L^{2}(\mathbb{P})} \leq 2\left(A_{4, n, N}+A_{5, n, N}\right) .
\end{aligned}
$$

Thus, we conclude that

$$
\left|\mathbb{E}\left[h\left(\sum_{m=q}^{N} \sigma_{m} Y_{m}\right)-h\left(S_{n, N}\right)\right]\right| \leq\left\|h^{\prime \prime}\right\|_{\infty}\left(A_{1, n}+A_{3, n, N}+A_{4, n, N}+A_{5, n, N}\right),
$$

which finishes this step.

(d) Putting things together. We have the inequality

$$
\begin{aligned}
\left|\mathbb{E}\left[h\left(S_{n}\right)\right]-\mathbb{E}[h(S)]\right| \leq & \left|\mathbb{E}\left[h\left(S_{n}\right)\right]-\mathbb{E}\left[h\left(S_{n, N}\right)\right]\right|+\left|\mathbb{E}\left[h\left(S_{n, N}\right)\right]-\mathbb{E}\left[h\left(Z_{N}\right)\right]\right| \\
& +\left|\mathbb{E}\left[h\left(Z_{N}\right)\right]-\mathbb{E}[h(S)]\right| .
\end{aligned}
$$

We are left with the derivation of a bound for the third term. By using (3.41) in the special case $F=Z_{N}$, we deduce that

$$
\begin{aligned}
\left|\mathbb{E}\left[h\left(Z_{N}\right)\right]-\mathbb{E}[h(S)]\right| & \leq \frac{1}{2}\left\|h^{\prime \prime}\right\|_{\infty} \sum_{m=N+1}^{\infty} \sigma_{m}^{2} \\
& \leq \frac{2 H+d^{q} \theta}{2}\left\|h^{\prime \prime}\right\|_{\infty} \sum_{m=N+1}^{\infty} \mathbb{E}\left[f_{m}^{2}\left(X_{1}\right)\right],
\end{aligned}
$$

where the last inequality is deduced by Lemma 4.1. The latter is smaller than $\frac{1}{4}\left\|h^{\prime \prime}\right\|_{\infty} A_{2, N}$, which together with (4.46)-(4.48) completes the proof of Theorem 2.1-(1).

\subsection{Proof of Theorem 2.1-(2)}

Take $h$ Lipschitz and write the inequality (4.49). Like for (4.46), standard computations yield

$$
\begin{aligned}
\left|\mathbb{E}\left[h\left(S_{n}\right)\right]-\mathbb{E}\left[h\left(S_{n, N}\right)\right]\right| \leq & \left\|h^{\prime}\right\|_{\infty}\left\|S_{n}-S_{n, N}\right\|_{L^{2}(\mathbb{P})} \leq\left\|h^{\prime}\right\|_{\infty}\left(2 K+d^{q} \theta\right)^{1 / 2} \\
& \times \sqrt{\sum_{m=N+1}^{\infty}\left\|f_{m}\left(X_{1}\right)\right\|_{L^{2}(\mathbb{P})}^{2}} .
\end{aligned}
$$


By applying inequality (3.40) in the case $F=S_{n, N}, Z=Z_{N}$, one has that

$$
\begin{aligned}
\left|\mathbb{E}\left[h\left(S_{n, N}\right)\right]-\mathbb{E}\left[h\left(Z_{N}\right)\right]\right| \leq & \frac{\left\|h^{\prime}\right\|_{\infty}}{\left(\sum_{m=q}^{N} \sigma_{m}^{2}\right)^{1 / 2}} \\
& \times \sum_{p, s=q}^{N}\left\|\delta_{p s} \sigma_{p}^{2}-s^{-1}\left\langle D I_{p}\left(g_{p}^{n}\right), D I_{s}\left(g_{s}^{n}\right)\right\rangle_{\mathfrak{H}}\right\|_{L^{2}(\mathbb{P})} \\
\leq & \frac{\left\|h^{\prime}\right\|_{\infty}}{\left(\sum_{m=q}^{N} \sigma_{m}^{2}\right)^{1 / 2}} 2\left(A_{1, n}+A_{3, n, N}+A_{4, n, N}+A_{5, n, N}\right),
\end{aligned}
$$

where the last inequality is obtained by reasoning as in Part (c) of Section 4.2. Finally, an application of inequality (3.42) in the case $F=Z_{N}$ yields (

$$
\left|\mathbb{E}\left[h\left(Z_{N}\right)\right]-\mathbb{E}[h(S)]\right| \leq \frac{\left\|h^{\prime}\right\|_{\infty}}{\sigma} \sum_{m=N+1}^{\infty} \sigma_{m}^{2} \leq \frac{\left\|h^{\prime}\right\|_{\infty}}{2 \sigma} A_{2, N} .
$$

Putting the above estimates together yields the desired conclusion.

\subsection{Proof of Theorem 2.1-(3)}

From [11, Theorem 3.1], one can deduce that

$$
d_{K o l}\left(S_{n}, S\right) \leq 2 \sqrt{d_{W}\left(S_{n} / \sigma, S / \sigma\right)} .
$$

Hence, we get the desired conclusion by combining this inequality with (2.20).

\section{Acknowledgement}

We warmly thank Arnaud Guillin for pointing out to us an inaccuracy in a previous version.

\section{References}

[1] V.V. Anh, N.N. Leonenko, Renormalization and homogenization of fractional diffusion equations with random data, Probab. Theory Related Fields 124 (3) (2002) 381-408.

[2] M.A. Arcones, Limit theorems for nonlinear functionals of a stationary Gaussian sequence of vectors, Ann. Probab. 22 (4) (1994) 2242-2274.

[3] O.E. Barndorff-Nielsen, J.M. Corcuera, M. Podolskij, Power variation for Gaussian processes with stationary increments, Stochastic Process Appl. 119 (2009) 1845-1865.

[4] O.E. Barndorff-Nielsen, J.M. Corcuera, M. Podolskij, Multipower variation for Brownian semi-stationary processes, Bernoulli (2009) (in press).

[5] L. Beghin, V.P. Knopova, N.N. Leonenko, E. Orsingher, Gaussian limiting behavior of the rescaled solution to the linear Korteweg-de Vries equation with random initial conditions, J. Stat. Phys. 99 (3-4) (2000) 769-781.

[6] N.H. Bingham, C.M. Goldie, J.L. Teugels, Regular Variation, Cambridge University Press, 1987.

[7] J.-C. Breton, I. Nourdin, Error bounds on the non-normal approximation of Hermite power variations of fractional Brownian motion, Electron. Comm. Probab. 13 (2008) 482-493 (electronic).

[8] P. Breuer, P. Major, Central limit theorems for nonlinear functionals of Gaussian fields, J. Multivariate Anal. 13 (3) (1983) 425-441.

[9] C. Berzin, J. León, Estimating the Hurst parameter, Stat. Inference Stoch. Process. 10 (1) (2007) 49-73. 
[10] B. Buchmann, N.-H. Chan, Integrated functionals of normal and fractional processes, Ann. Appl. Probab. 19 (1) (2009) 49-70.

[11] L. Chen, Q.-M. Shao, Stein's method for normal approximation, in: An Introduction to Stein's Method, in: Lect. Notes Ser. Inst. Math. Sci. Natl. Univ. Singap., vol. 4, Singapore Univ. Press, Singapore, 2005, pp. 1-59.

[12] J.-F. Coeurjolly, Identification of multifractional Brownian motion, Bernoulli 11 (6) (2005) 987-1008.

[13] J.-F. Coeurjolly, Hurst exponent estimation of locally self-similar Gaussian processes using sample quantiles, Ann. Statist. 36 (3) (2008) 1404-1434.

[14] J.M. Corcuera, D. Nualart, J.H.C. Woerner, Power variation of some integral fractional processes, Bernoulli 12 (4) (2006) 713-735.

[15] R.L. Dobrushin, P. Major, Non-central limit theorems for nonlinear functionals of Gaussian fields, Z. Wahrscheinlichkeitstheor. Verwandte Geb. 50 (1) (1979) 27-52.

[16] R.M. Dudley, Real Analysis and Probability, 2nd ed., Cambridge University Press, Cambridge, 2003.

[17] L. Giraitis, D. Surgailis, CLT and other limit theorems for functionals of Gaussian processes, Z. Wahrscheinlichkeitstheor. Verwandte Geb. 70 (1985) 191-212.

[18] S. Janson, Gaussian Hilbert Spaces, Cambridge University Press, Cambridge, 1997.

[19] M.F. Kratz, J.R. León, Central limit theorems for level functionals of stationary Gaussian processes and fields, J. Theoret. Probab. 14 (3) (2001) 639-672.

[20] J. León, C. Ludeña, Limits for weighted $p$-variations and likewise functionals of fractional diffusions with drift, Stochastic Process. Appl. 117 (3) (2007) 271-296.

[21] C. Ludeña, $L^{p}$-variations for multifractal fractional random walks, Ann. Appl. Probab. 18 (3) (2008) $1138-1163$.

[22] D. Marinucci, The empirical process for bivariate sequences with long memory, Stat. Inference Stoch. Process. 8 (2) (2002) 205-223.

[23] D. Marinucci, A Central limit theorem and higher order results for the angular bispectrum, Probab. Theory Related Fields 141 (2007) 389-409.

[24] I. Nourdin, D. Nualart, C.A. Tudor, Central and non-central limit theorems for weighted power variations of fractional Brownian motion, Ann. Inst. H. Poincaré Probab. Statist. 46 (4) (2008) 1055-1079.

[25] I. Nourdin, G. Peccati, Stein's method on Wiener chaos, Probab. Theory Related Fields 145 (1) (2009) $75-118$.

[26] I. Nourdin, G. Peccati, Stein's method and exact Berry-Esseen asymptotics for functionals of Gaussian fields, Ann. Probab. 37 (6) (2010) 2231-2261.

[27] I. Nourdin, G. Peccati, G. Reinert, Invariance principles for homogeneous sums: universality of Gaussian Wiener chaos, Ann. Probab. 38 (5) (2010) 1947-1985.

[28] D. Nualart, The Malliavin Calculus and Related Topics, 2nd ed., Springer, Berlin, 2006.

[29] G. Peccati, M.S. Taqqu, Wiener Chaos: Moments, Cumulants and Diagrams, Springer, Berlin, 2010.

[30] G. Peccati, C. Zheng, Multi-dimensional Gaussian fluctuations on the Poisson space, Electron. J. Probab. 15 (48) (2010) 1487-1527.

[31] M. Rosenblatt, Independence and dependence, in: Proc. 4th Berkeley Sympos. Math. Statist. and Prob., vol. II, Univ. California Press, Berkeley, Calif, 1961, pp. 431-443.

[32] M. Sodin, B. Tsirelson, Random complex zeroes. I. Asymptotic normality, Israel J. Math. 144 (2004) $125-149$.

[33] T.-C. Sun, Some further results on central limit theorems for nonlinear functions of a normal stationary process, J. Math. Mech. 14 (1965) 71-85.

[34] D. Surgailis, CLTs for polynomials of linear sequences: diagram formulae with applications, in: Long Range Dependence, Birkhäuser, Basel, 2000, pp. 111-128.

[35] M. Talagrand, Spin Glasses: a Challenge for Mathematicians. Cavity and Mean Fields, Springer, Berlin, 2003.

[36] M.S. Taqqu, Weak convergence to fractional Brownian motion and to the Rosenblatt process, Z. Wahrscheinlichkeitstheor. Verwandte Geb. 31 (1975) 287-302.

[37] M.S. Taqqu, Convergence of integrated processes of arbitrary Hermite rank, Z. Wahrscheinlichkeitstheor. Verwandte Geb. 50 (1) (1979) 53-83.

[38] L. Wang, Asymptotics of statistical estimates in stochastic programming problems with long-range dependent samples, Math. Methods Oper. Res. 55 (1) (2002) 37-54. 